\title{
Real-Analytic Non-Integrable Functions on the Plane with Equal Iterated Integrals
}

\author{
Luis Bernal-González, María del Carmen Calderón-Moreno®, and \\ Andreas Jung
}

\begin{abstract}
In this note, a vector space of real-analytic functions on the plane is explicitly constructed such that all its nonzero functions are nonintegrable but yet their two iterated integrals exist as real numbers and coincide. Moreover, it is shown that this vector space is dense in the space of all real continuous functions on the plane endowed with the compactopen topology.
\end{abstract}

Mathematics Subject Classification. (MSC 2020) 26E05, 26B15, 28A35, $46 \mathrm{~B} 87$.

Keywords. Fubini's theorem, Real analytic functions, Iterated integrals.

\section{Introduction}

Fubini's theorem asserts that if a real function $f$, which is defined on a measure space $X \times Y$ that is the product of two $\sigma$-finite product spaces, is integrable, then its two iterated integrals exist as real numbers and coincide, and in fact their common value is the integral of $f$ on the product space; see e.g. [8, Chapter 17]. Throughout this paper we shall consider the real line $\mathbb{R}$ as endowed with the Lebesgue measure $d x$, so that the plane $\mathbb{R}^{2}=\mathbb{R} \times \mathbb{R}$ is equipped with the bidimensional Lebesgue measure $d x d y$, which is nothing but the product measure of $d x$ with itself.

This research has been supported by the Plan Andaluz de Investigación de la Junta de Andalucía FQM-127 Grant P20-00637 and by MCINN Grant PGC2018-098474-B-C21. 
Of course, Fubini's theorem is only a sufficient criterion, so that it is not too difficult to provide examples of non-integrable functions $\mathbb{R}^{2} \rightarrow \mathbb{R}$ such that the iterated integrals exist as real numbers and share the same value. For instance, if $I_{k}:=(k, k+1)(k \in \mathbb{N}:=\{1,2, \ldots\})$, then the function $f: \mathbb{R}^{2} \rightarrow \mathbb{R}$ given by

$$
f(x, y)=\left\{\begin{array}{l}
1 \quad \text { if }(x, y) \in I_{1}^{2} \cup \bigcup_{n \in \mathbb{N}}\left[\left(I_{2 n} \times I_{2 n+1}\right) \cup\left(I_{2 n+1} \times I_{2 n}\right)\right] \\
-1 \text { if }(x, y) \in \bigcup_{n \in \mathbb{N}}\left[\left(I_{2 n} \times I_{2 n-1}\right) \cup\left(I_{2 n-1} \times I_{2 n}\right)\right] \\
0 \quad \text { otherwise }
\end{array}\right.
$$

is Lebesgue-measurable and satisfies

$$
\int_{\mathbb{R}}\left(\int_{\mathbb{R}} f(x, y) d x\right) d y=\int_{\mathbb{R}} 0 d y=0=\int_{\mathbb{R}} 0 d x=\int_{\mathbb{R}}\left(\int_{\mathbb{R}} f(x, y) d y\right) d x,
$$

but it is not integrable on $\mathbb{R}^{2}$ because, as $|f|=1$ on a set of infinite area, we have $\iint_{\mathbb{R}^{2}}|f(x, y)| d x d y=+\infty$. In the much more general setting of the product $X \times Y$ of two measurable spaces, the existence of measurable functions $X \times Y \rightarrow \mathbb{R}$ satisfying analogous properties -as well as the size of the set of them- is analyzed in [3].

A natural question is whether such "pseudo-Fubini functions" $f$ can be found to be continuous on $\mathbb{R}^{2}$. This is, again, not too difficult: for instance, just replace " 1 " and " -1 " in (1) by, respectively, $\varphi_{m}(x) \varphi_{p}(y)$ and $-\varphi_{m}(x) \varphi_{p}(y)$ on each product $I_{m} \times I_{p}(m, p \in \mathbb{N})$, where

$$
\varphi_{k}(t):= \begin{cases}t-k & \text { if } t \in\left(k, k+\frac{1}{2}\right] \\ k+1-t & \text { if } t \in\left(k+\frac{1}{2}, k+1\right)\end{cases}
$$

for every $k \in \mathbb{N}$.

Going one step further, we may wonder whether $f$ can even be chosen to live in the class $C^{\infty}\left(\mathbb{R}^{2}\right)$ of infinitely differentiable functions $\mathbb{R}^{2} \rightarrow \mathbb{R}$. The answer is, again, affirmative: the construction of such an $f$ is as in the preceding paragraph, with the sole exception that each function $\varphi_{k}:(k, k+1) \rightarrow \mathbb{R}$ $(k \in \mathbb{N})$ in $(2)$ is this time given by

$$
\varphi_{k}(t)=e^{\frac{1}{(t-k)(t-k-1)}} .
$$

Then the following questions arises: Are there real-analytic pseudo-Fubini functions on $\mathbb{R}^{2}$ ? Recall, if $\Omega$ is an open subset of $\mathbb{R}^{2}$, then a function $f: \Omega \rightarrow$ $\mathbb{R}$ is said to be real-analytic on $\Omega$ whenever, given $\left(x_{0}, y_{0}\right) \in \Omega$, there are an neighborhood $U$ of $\left(x_{0}, y_{0}\right)$ with $U \subset \Omega$ and a double sequence $\left\{a_{j k}\right\}_{j, k \geq 0} \subset \mathbb{R}$ such that $f(x, y)=\sum_{j, k \geq 0} a_{j k}\left(x-x_{0}\right)^{j}\left(y-y_{0}\right)^{k}$ for every $(x, y) \in U$, the convergence being absolute. The set of all such functions will be denoted by $C^{\omega}(\Omega)$. Note that, due to the Analytic Continuation Principle, the piece-bypiece construction of the last three paragraphs is not possible in the analytic case. The reader is referred to [4, Chap. 4] for background on real or complex analytic functions of several variables. 
The aim of this short note is to show that not only such real-analytic functions exist but also that there is a plethora of them. Specifically, we shall construct a vector space of real-analytic functions in the plane all of whose nonzero members are non-integrable but satisfy that both iterated integrals exist as real numbers and share the same value. Moreover, this vector space has the dimension of the continuum and is dense in the space of all continuous functions in the plane when endowed with the topology of uniform convergence on compacta. The problem of replacing the class of analytic functions by the more stringent one of entire functions is also considered.

\section{Construction of a Special Class of Real-Analytic Functions}

In our construction we shall consider the vector space $C(\Omega)$ of continuous functions $\Omega \rightarrow \mathbb{R}$, where $\Omega$ is a nonempty open subset of $\mathbb{R}^{2}$. Note that $C^{\omega}(\Omega) \subset C^{\infty}(\Omega) \subset C(\Omega)$. Recall that $C(\Omega)$ becomes a separable complete metrizable topological vector space when it is endowed with the compact-open topology, that is, the topology of uniform convergence on compact subsets of $\Omega$ (see e.g. [6, Chapter 2]). For instance, a translation-invariant distance generating this topology is given by

$$
d(f, g):=\sup _{n \in \mathbb{N}} \min \left\{1 / n, \sup _{B_{n}}|f-g|\right\}
$$

where $B_{n}$ is the Euclidean closed ball centered at the origin with radius $n$.

It is also well-known (an easy proof follows, for instance, from the Baire category theorem) that $\operatorname{dim} C(\Omega)=\mathfrak{c}$, the cardinality of the continuum. Hence, $\mathfrak{c}$ is the maximal value that one can expect for the dimension of a vector subspace of $C(\Omega)$.

Our results concerning pseudo-Fubini analytic functions are collected in the next theorem. Note that it tells us that there is a plethora of such functions, in both topological and algebraic senses.

Theorem 1. There is a family $\mathcal{A}$ of functions $\mathbb{R}^{2} \rightarrow \mathbb{R}$ enjoying the following properties:

(a) $\mathcal{A} \subset C^{\omega}\left(\mathbb{R}^{2}\right)$.

(b) For every $f \in \mathcal{A}$ both iterated Lebesgue integrals $\int_{\mathbb{R}}\left(\int_{\mathbb{R}} f(x, y) d x\right) d y$, $\int_{\mathbb{R}}\left(\int_{\mathbb{R}} f(x, y) d y\right) d x$ exist as real numbers and have the same value.

(c) No nonzero function in $\mathcal{A}$ is Lebesgue-integrable on $\mathbb{R}^{2}$.

(d) $\mathcal{A}$ is a $\mathfrak{c}$-dimensional vector space.

(e) $\mathcal{A}$ is dense in $C\left(\mathbb{R}^{2}\right)$. 
Proof. Our first task is to locate an analytic pseudo-Fubini function on $\mathbb{R}^{2}$. We start with the function

$$
f:(x, y) \in(0,1)^{2} \longmapsto \frac{x-y}{(x+y)^{3}} \in \mathbb{R} .
$$

Clearly, $f \in C^{\omega}\left((0,1)^{2}\right)$. Now, we practice a number of elementary calculations. If we fix $y \in(0,1)$ then we have

$$
\begin{aligned}
& \int_{0}^{1} f(x, y) d x=\int_{0}^{1} \frac{(x+y)-2 y}{(x+y)^{3}} d x=\int_{0}^{1}\left((x+y)^{-2}-2 y(x+y)^{-3}\right) d x \\
& =\left[-(x+y)^{-1}+y(x+y)^{-2}\right]_{x=0}^{x=1}=\frac{-1}{1+y}+\frac{1}{y}+\frac{y}{(1+y)^{2}}-\frac{1}{y}=\frac{-1}{(1+y)^{2}} .
\end{aligned}
$$

Therefore

$$
\int_{0}^{1}\left(\int_{0}^{1} f(x, y) d x\right) d y=\int_{0}^{1} \frac{-1}{(1+y)^{2}} d y=\left[\frac{1}{1+y}\right]_{y=0}^{y=1}=\frac{1}{2}-\frac{1}{1}=-\frac{1}{2} .
$$

Since $f(x, y)=-f(y, x)$ for all $(x, y) \in(0,1)^{2}$, by exchanging the roles of $x$ and $y$ we obtain

$$
\int_{0}^{1}\left(\int_{0}^{1} f(x, y) d y\right) d x=-\int_{0}^{1}\left(\int_{0}^{1} f(y, x) d y\right) d x=-\frac{-1}{2}=\frac{1}{2} .
$$

Next, we consider the function $g:(0,1)^{2} \longrightarrow \mathbb{R}$ defined as

$$
g(x, y)=f(x, y)-f(1-x, 1-y)=(x-y) \cdot\left\{(x+y)^{-3}+(2-x-y)^{-3}\right\} .
$$

Again, $g \in C^{\omega}\left((0,1)^{2}\right)$. Let $\Phi(y):=\int_{0}^{1} f(x, y) d x$ for $y \in(0,1)$. Now, observe that by applying successively the changes of variables $x \mapsto 1-x$ and $y \mapsto 1-y$ we get

$$
\begin{gathered}
\int_{0}^{1}\left(\int_{0}^{1} f(1-x, 1-y) d x\right) d y=\int_{0}^{1}\left(\int_{0}^{1} f(x, 1-y) d x\right) d y \\
=\int_{0}^{1} \Phi(1-y) d y=\int_{0}^{1} \Phi(y) d y=\int_{0}^{1}\left(\int_{0}^{1} f(x, y) d x\right) d y
\end{gathered}
$$

It follows from (3) that

$$
\int_{0}^{1}\left(\int_{0}^{1} g(x, y) d x\right) d y=0
$$

and, analogously, 


$$
\int_{0}^{1}\left(\int_{0}^{1} g(x, y) d y\right) d x=0 .
$$

Next, let us observe that the mappings $x \in(0,1) \longmapsto x^{\alpha} \in(0,1)(\alpha \geq 1)$ and $\varphi: s \in \mathbb{R} \longmapsto \frac{1}{2}+\frac{1}{\pi} \cdot \arctan s \in(0,1)$ are analytic diffeomorphisms. Since analyticity is stable under composition, we get that each mapping

$$
\varphi_{\alpha}: s \in \mathbb{R} \longmapsto(\varphi(s))^{\alpha} \in(0,1) \quad(\alpha \geq 1)
$$

is an analytic diffeomorphism. In order to use the theorem of change of variables for one-dimensional or multidimensional integrals (see, e.g., [5, Chapter $3])$, we remark that $\varphi_{\alpha}^{\prime}(s)=\frac{\alpha \cdot \varphi_{\alpha-1}(s)}{\pi \cdot\left(1+s^{2}\right)}(s \in \mathbb{R})$, and that the mapping

$$
\Psi:(s, t) \in \mathbb{R}^{2} \longmapsto(\varphi(s), \varphi(t)) \in(0,1) \times(0,1)
$$

is a diffeomorphism whose Jacobian determinant is $\operatorname{det} J_{\Psi}(s, t)=\frac{1}{\pi^{2}\left(1+s^{2}\right)\left(1+t^{2}\right)}$.

For every fixed $\alpha \in[1,+\infty)$, define the function $f_{\alpha}: \mathbb{R}^{2} \rightarrow \mathbb{R}$ by

$$
f_{\alpha}(s, t):=\frac{\varphi_{\alpha-1}(s) \varphi_{\alpha-1}(t) g\left(\varphi_{\alpha}(s), \varphi_{\alpha}(t)\right)}{\left(1+s^{2}\right)\left(1+t^{2}\right)} .
$$

We shall need for our purposes a subset of integrable real-analytic functions that is dense in $C\left(\mathbb{R}^{2}\right)$. Consider the countable set

$\mathcal{D}:=\left\{P \cdot G_{1}: P\right.$ is a polynomial of two real variables with coefficients in $\left.\mathbb{Q}\right\}$, where $\mathbb{Q}$ is the set of rational numbers and $G_{1}$ is the Gaussian function $G_{1}(x, y)=e^{-x^{2}-y^{2}}$, that is integrable on the plane (with integral equal to $\pi)$ together with every function $G_{\alpha}(x, y):=e^{-\alpha\left(x^{2}+y^{2}\right)}$ with $\alpha>0$. Plainly, $\mathcal{D} \subset C^{\omega}\left(\mathbb{R}^{2}\right)$.

On the one hand, each function $h:=P \cdot G_{1} \in \mathcal{D}$ is integrable. Indeed, since $P$ is a polynomial, there are a constant $M \in(0,+\infty)$ and an $N \in \mathbb{N}$ such that $|P(x, y)| \leq M\left(1+\left(x^{2}+y^{2}\right)^{N}\right)$ for all $(x, y) \in \mathbb{R}^{2}$. And since $\lim _{t \rightarrow+\infty} \frac{M\left(1+t^{N}\right)}{e^{t / 2}}=0$, we derive the existence of a constant $\gamma \in(0,+\infty)$ such that $|P(x, y)| \leq \gamma \cdot e^{\frac{x^{2}+y^{2}}{2}}$ on $\mathbb{R}^{2}$, and therefore $|h(x, y)| \leq \gamma \cdot e^{\frac{-x^{2}-y^{2}}{2}}$ on $\mathbb{R}^{2}$. The comparison principle yields the integrability of $h$. In particular, Fubini's theorem implies that

$$
\int_{\mathbb{R}}\left(\int_{\mathbb{R}} h(x, y) d x\right) d y=\iint_{\mathbb{R}^{2}} h(x, y) d x d y=\int_{\mathbb{R}}\left(\int_{\mathbb{R}} h(x, y) d y\right) d x
$$

for all $h \in \mathcal{D}$.

On the other hand, it follows from the Stone-Weierstrass theorem in its version for completely regular spaces (see [7, Theorem 16.5.7]; note that, since $\mathbb{R}^{2}$ is metrizable, it is completely regular), that the algebra generated by the functions $(x, y) \mapsto x,(x, y) \mapsto y$ is dense in $C\left(\mathbb{R}^{2}\right)$. But this algebra is the set of all polynomials of two real variables. Fix a function $F \in C\left(\mathbb{R}^{2}\right)$, as well as an $\varepsilon>0$ and a compact set $K \subset \mathbb{R}^{2}$. Select a polynomial $P_{0}$ with $\left|P_{0}-\frac{F}{G_{1}}\right|<\frac{\varepsilon}{2}$ on $K$. Now, pick a polynomial $P$ having rational coefficients such that $\left|P-P_{0}\right|<\frac{\varepsilon}{2}$ on $K$. This together with the triangle inequality and 
the fact $\left|G_{1}\right| \leq 1$ yields $|h-F|<\varepsilon$ on $K$, where $h:=P \cdot G_{1} \in \mathcal{D}$. We have proved the denseness of $\mathcal{D}$ in $C\left(\mathbb{R}^{2}\right)$.

Our next step is to construct the desired family $\mathcal{A}$ satisfying properties (a) to (e). With this aim, put $\mathcal{D}=\left\{h_{n}: n \in \mathbb{N}\right\}$, which is possible because of the countability of $\mathcal{D}$. Select any sequence $\left\{c_{n}\right\}_{n \in \mathbb{N}}$ of pairwise different points of $[1,2]$. For each $n \in \mathbb{N}$, the fact that $C\left(\mathbb{R}^{2}\right)$ is a topological vector space implies the existence of a constant $\mu_{n}>0$ such that $d\left(\mu_{n} f_{c_{n}}, 0\right)<1 / n$, where $f_{c_{n}}$ is defined by (6). Now, we define

$$
\mathcal{A}:=\operatorname{span}\left(\left\{h_{n}+\mu_{n} f_{c_{n}}: n \in \mathbb{N}\right\} \cup\left\{f_{\alpha}: \alpha>2\right\}\right) .
$$

Observe first that $\mathcal{A}$ is a vector subspace of $C\left(\mathbb{R}^{2}\right)$. In addition, each $f_{\alpha}$ with $\alpha \geq 1$ and each $h_{n}$ is analytic, which yields $\mathcal{A} \subset C^{\omega}\left(\mathbb{R}^{2}\right)$, that is (a). Moreover, since $\left\{h_{n}\right\}_{n \in \mathbb{N}}$ is dense in $C\left(\mathbb{R}^{2}\right)$ and

$$
0 \leq d\left(h_{n}, h_{n}+\mu_{n} f_{c_{n}}\right)=d\left(\mu_{n} f_{c_{n}}, 0\right)<1 / n \longrightarrow 0 \quad(n \rightarrow \infty),
$$

one easily derives that the set $\left\{h_{n}+\mu_{n} f_{c_{n}}\right\}_{n \in \mathbb{N}}$ is also dense in $C\left(\mathbb{R}^{2}\right)$. But $\mathcal{A} \supset\left\{h_{n}+\mu_{n} f_{c_{n}}\right\}_{n \in \mathbb{N}}$, which shows that $\mathcal{A}$ is dense in $C\left(\mathbb{R}^{2}\right)$, that is (e).

Now, our aim is to prove that any nonzero function $F \in \mathcal{A}$ is not integrable on $\mathbb{R}^{2}$. By (8), for such a function there are respective finite subsets $F_{1} \subset \mathbb{N}, F_{2} \subset(2,+\infty)$ with $F_{1} \cup F_{2} \neq \varnothing$ as well as scalars $\beta_{i}, \delta_{j} \in \mathbb{R} \backslash\{0\}$ $\left(i \in F_{1}, j \in F_{2}\right)$ satisfying $F=\sum_{i \in F_{1}} \beta_{i}\left(h_{i}+\mu_{i} f_{c_{i}}\right)+\sum_{j \in F_{2}} \delta_{j} f_{j}$, where any of the sums $\sum_{i \in F_{1}}$ or $\sum_{j \in F_{2}}$ is understood as 0 if, respectively, $F_{1}=\varnothing$ or $F_{2}=\varnothing$. After relabeling, we get

$$
F=h+\sum_{i=1}^{p} \lambda_{i} f_{\alpha_{i}}
$$

for certain $p \in \mathbb{N},\left\{\lambda_{1}, \ldots, \lambda_{p}\right\} \subset \mathbb{R} \backslash\{0\}$ and $\left\{\alpha_{1}<\alpha_{2}<\cdots<\alpha_{p}\right\} \subset$ $[1,+\infty)$, where $h \in \mathcal{D}$. Assume, by way of contradiction, that $F$ is integrable on $\mathbb{R}^{2}$. Since $h$ is integrable, this would yield the integrability of the function $G:=\sum_{i=1}^{p} \lambda_{i} f_{\alpha_{i}}$. According to (6), we have

$$
G(s, t)=\sum_{i=1}^{p} \lambda_{i} \cdot \frac{\varphi_{\alpha_{i}-1}(s) \varphi_{\alpha_{i}-1}(t) g\left(\varphi_{\alpha_{i}}(s), \varphi_{\alpha_{i}}(t)\right)}{\left(1+s^{2}\right)\left(1+t^{2}\right)},
$$

where $\varphi_{\gamma}(s)=\varphi(s)^{\gamma}=\left(\frac{1}{2}+\frac{1}{\pi} \cdot \arctan s\right)^{\gamma}$ for all $\gamma \geq 0$ and all $s \in \mathbb{R}$. If we consider the diffeomorphism $\Psi(s, t)=(\varphi(s), \varphi(t))$, the change of variables theorem leads us to the integrability on $(0,1)^{2}$ of the function $\widetilde{G}:(0,1)^{2} \rightarrow \mathbb{R}$ given by

$$
\widetilde{G}(x, y)=\sum_{i=1}^{p} \lambda_{i} \cdot x^{\alpha_{i}-1} y^{\alpha_{i}-1} \cdot g\left(x^{\alpha_{i}}, y^{\alpha_{i}}\right),
$$


which, according to (3), can be written as

$$
\begin{aligned}
& \widetilde{G}(x, y)=\sum_{i=1}^{p} \lambda_{i} \cdot x^{\alpha_{i}-1} y^{\alpha_{i}-1} \\
& \quad \cdot\left(x^{\alpha_{i}}-y^{\alpha_{i}}\right) \cdot\left\{\left(x^{\alpha_{i}}+y^{\alpha_{i}}\right)^{-3}+\left(2-x^{\alpha_{i}}-y^{\alpha_{i}}\right)^{-3}\right\} .
\end{aligned}
$$

Trivially, $\widetilde{G}$ would also be integrable on the square $(0,1 / 2)^{2}$. But, since each function $\frac{x^{\alpha_{i}-1} y^{\alpha_{i}-1}\left(x^{\alpha_{i}}-y^{\alpha_{i}}\right)}{\left(2-x^{\alpha_{i}}-y^{\alpha_{i}}\right)^{3}}$ is continuous on the closed square $[0,1 / 2]^{2}$, it is integrable on $(0,1 / 2)^{2}$, and this implies that the function

$$
H(x, y):=\sum_{i=1}^{p} \lambda_{i} \cdot \frac{x^{\alpha_{i}-1} y^{\alpha_{i}-1}\left(x^{\alpha_{i}}-y^{\alpha_{i}}\right)}{\left(x^{\alpha_{i}}+y^{\alpha_{i}}\right)^{3}}
$$

is also integrable on $(0,1 / 2)^{2}$.

For every $c \in(0,1 / 2)$ we define the triangular set

$$
S_{c}:=\left\{(x, y) \in \mathbb{R}^{2}: 0<x<\frac{1}{2} \text { and } c x<y<2 c x\right\} .
$$

Observe that $S_{c}$ is open (so Lebesgue-measurable) and $S \subset(0,1 / 2)^{2}$. Then $H$ is integrable on $S_{c}$. Letting $\psi_{\alpha}(x, y):=\frac{x^{\alpha-1} y^{\alpha-1}\left(x^{\alpha}-y^{\alpha}\right)}{\left(x^{\alpha}+y^{\alpha}\right)^{3}}$ we are allowed to write

$$
H=\sum_{i=1}^{p} \lambda_{i} \cdot \psi_{\alpha_{i}}
$$

Moreover, for every $(x, y) \in S_{c}$ and every $\alpha \geq 1$, it is easy to check that

$$
0<\frac{1}{8} \cdot c^{\alpha-1}\left(1-2^{\alpha} c^{\alpha}\right) \cdot x^{-2} \leq \psi_{\alpha}(x, y) \leq 2^{\alpha-1} c^{\alpha-1} \cdot x^{-2}
$$

We assume that $p>1$ (the case $p=1$ is easier to deal with). Let us define the functions $\mu, \nu:(0,1 / 2) \rightarrow \mathbb{R}$ by

$$
\mu(c)=\left|\lambda_{1}\right| \cdot \frac{1}{8} \cdot c^{\alpha_{1}-1}\left(1-2^{\alpha_{1}} c^{\alpha_{1}}\right) \text { and } \nu(c)=\sum_{j=2}^{p} \frac{\left|\lambda_{j}\right| 2^{\alpha_{j}+2} c^{\alpha_{j}-\alpha_{1}}}{\left|\lambda_{1}\right|\left(1-2^{\alpha_{1}} c^{\alpha_{1}}\right)} .
$$

Observe that $\mu(c)>0$ for all $c \in(0,1 / 2)$. In addition, it follows from $0<$ $\alpha_{1}<\alpha_{j}(j=2, \ldots, p)$ that

$$
\lim _{c \rightarrow 0^{+}} \nu(c)=0
$$


In particular, we can select a value $c \in(0,1 / 2)$ such that $\nu(c)<\frac{1}{2}$. From (9), (10) and the triangle inequality we obtain that for every $(x, y) \in S_{c}$ (with $c$ chosen as before) the following holds:

$$
\begin{aligned}
|H(x, y)| & =\left|\sum_{i=1}^{p} \lambda_{i} \cdot \psi_{\alpha_{i}}(x, y)\right| \\
& \geq\left|\lambda_{1}\right| \cdot \psi_{\alpha_{1}}(x, y) \cdot\left(1-\sum_{i=2}^{p} \frac{\left|\lambda_{i}\right| \cdot \psi_{\alpha_{i}}(x, y)}{\left|\lambda_{1}\right| \cdot \psi_{\alpha_{1}}(x, y)}\right) \\
& \geq \mu(c) \cdot x^{-2} \cdot(1-\nu(c)) \geq \frac{\mu(c)}{2} \cdot x^{-2} .
\end{aligned}
$$

Recall that the conclusion of Fubini's theorem holds for nonnegative measurable functions. Therefore

$$
\begin{aligned}
\iint_{S_{c}}|H(x, y)| d x d y & \geq \iint_{S_{c}} \frac{\mu(c)}{2} \cdot x^{-2} d x d y=\frac{\mu(c)}{2} \cdot \int_{0}^{1 / 2}\left(\int_{c x}^{2 c x} x^{-2} d y\right) d x \\
& =\frac{\mu(c)}{2} \cdot \int_{0}^{1 / 2} x^{-2}(2 c x-c x) d x \\
& =\frac{c \mu(c)}{2} \cdot \int_{0}^{1 / 2} x^{-1} d x=+\infty,
\end{aligned}
$$

and so $H$ is not integrable on $S_{c}$, which is absurd. This contradiction shows that $F$ is not integrable on $\mathbb{R}^{2}$, which is (c). Note that the same argument (taking $h=0$ and $\alpha_{i} \in(2,+\infty)$ ) yields the linear independence of the functions $f_{\alpha}(\alpha>2)$. But these functions belong to $\mathcal{A}$. Consequently, $\operatorname{dim}(\mathcal{A})=\mathfrak{c}$, that is $(d)$.

Concerning the property (b), consider a function $F \in \mathcal{A}$. Again, $F$ has the shape $F=h+\sum_{i=1}^{p} \lambda_{i} f_{\alpha_{i}}$ for certain $p \in \mathbb{N},\left\{\lambda_{1}, \ldots, \lambda_{p}\right\} \subset \mathbb{R}$, $\left\{\alpha_{1}, \alpha_{2}, \ldots, \alpha_{p}\right\} \subset[1,+\infty)$, and $h \in \mathcal{D}$. The $\lambda_{i}$ 's that are zero make no influence in the calculations, so that we can assume $\lambda_{i} \neq 0$ for all $i=1, \ldots, p$. Let us denote

$$
L(y):=\int_{0}^{1} g(x, y) d x \text { for } y \in(0,1) .
$$

It follows from (4), (5), (6), the linearity of the integral and the change of variable formula for integrals on $\mathbb{R}$ (twice applied) that both iterated integrals for $F$ exist as real numbers and

$$
\begin{aligned}
\int_{\mathbb{R}} & \left(\int_{\mathbb{R}} F(s, t) d s\right) d t=\int_{\mathbb{R}}\left(\int_{\mathbb{R}} h(s, t) d s\right) d t+\sum_{i=1}^{p} \lambda_{i} \int_{\mathbb{R}}\left(\int_{\mathbb{R}} f_{\alpha_{i}}(s, t) d s\right) d t \\
& =\int_{\mathbb{R}}\left(\int_{\mathbb{R}} h(s, t) d s\right) d t+\sum_{i=1}^{p} \frac{\lambda_{i} \pi^{2}}{\alpha_{i}^{2}} \int_{\mathbb{R}} \varphi_{\alpha_{i}}^{\prime}(t)\left(\int_{\mathbb{R}} \varphi_{\alpha_{i}}^{\prime}(s) g\left(\varphi_{\alpha_{i}}(s), \varphi_{\alpha_{i}}(t)\right) d s\right) d t
\end{aligned}
$$




$$
\begin{aligned}
& =\int_{\mathbb{R}}\left(\int_{\mathbb{R}} h(s, t) d s\right) d t+\sum_{i=1}^{p} \frac{\lambda_{i} \pi^{2}}{\alpha_{i}^{2}} \int_{\mathbb{R}} \varphi_{\alpha_{i}}^{\prime}(t)\left(\int_{0}^{1} g\left(x, \varphi_{\alpha_{i}}(t)\right) d x\right) d t \\
& =\int_{\mathbb{R}}\left(\int_{\mathbb{R}} h(s, t) d s\right) d t+\sum_{i=1}^{p} \frac{\lambda_{i} \pi^{2}}{\alpha_{i}^{2}} \int_{\mathbb{R}} \varphi_{\alpha_{i}}^{\prime}(t) L\left(\varphi_{\alpha_{i}}(t)\right) d t \\
& =\int_{\mathbb{R}}\left(\int_{\mathbb{R}} h(s, t) d s\right) d t+\sum_{i=1}^{p} \frac{\lambda_{i} \pi^{2}}{\alpha_{i}^{2}} \int_{0}^{1} L(y) d y \\
& =\int_{\mathbb{R}}\left(\int_{\mathbb{R}} h(s, t) d s\right) d t+\sum_{i=1}^{p} \frac{\lambda_{i} \pi^{2}}{\alpha_{i}^{2}} \int_{0}^{1}\left(\int_{0}^{1} g(x, y) d x\right) d y \\
& =\int_{\mathbb{R}}\left(\int_{\mathbb{R}} h(s, t) d s\right) d t+\sum_{i=1}^{p} \frac{\lambda_{i} \pi^{2}}{\alpha_{i}^{2}} \cdot 0=\int_{\mathbb{R}}\left(\int_{\mathbb{R}} h(s, t) d s\right) d t,
\end{aligned}
$$

and analogously,

$$
\int_{\mathbb{R}}\left(\int_{\mathbb{R}} F(s, t) d t\right) d s=\int_{\mathbb{R}}\left(\int_{\mathbb{R}} h(s, t) d t\right) d s .
$$

An application of $(7)$ gives $\int_{\mathbb{R}}\left(\int_{\mathbb{R}} F(s, t) d s\right) d t=\int_{\mathbb{R}}\left(\int_{\mathbb{R}} F(s, t) d t\right) d s$, which is (b). The theorem is proved.

\section{Final Remarks}

1. Under the terminology of the modern theory of lineability (see [2] for background), what has been proved in Theorem 1 is that the set of non-integrable real analytic functions on $\mathbb{R}^{2}$ having finite equal iterated integrals is maximal dense-lineable in $C\left(\mathbb{R}^{2}\right)$. A slight shortening of the proof could have been done by using [2, Theorem 7.3.1], but our approach has the virtue of being totally constructive.

2. The existence of real entire functions -that is, of functions $f: \mathbb{R}^{2} \rightarrow \mathbb{R}$ for which an absolutely convergent expansion $f(x, y)=\sum_{j, k \geq 0} a_{j k} x^{j} y^{k}$ is valid on the whole plane- that are Lebesgue-integrable is well known. The simplest instance of such functions is maybe $f(x, y)=e^{-x^{2}-y^{2}}$, which, incidentally, has been used in the proof of Theorem 1. Note that every entire function is in $C^{\omega}\left(\mathbb{R}^{2}\right)$. This raises in a natural way the problem -which we pose hereof existence of non-integrable real entire functions whose iterated Lebesgue integrals exist as real numbers and coincide. If the answer were affirmative, the study of the size of the set of such functions would also be interesting.

3. As a first step towards the solution of the problem posed in the preceding remark, we have been able to find a non-Lebesgue-integrable entire function $f: \mathbb{R}^{2} \rightarrow \mathbb{R}$ such that both iterated integrals do exist in the Riemann sense (but not in the Lebesgue one) and coincide. Indeed, consider the function $f(x, y):=\sin \left(x^{2}+y^{2}\right)$, which is entire because both functions $t \in \mathbb{R} \mapsto \sin t \in \mathbb{R}$ and $(x, y) \in \mathbb{R}^{2} \mapsto x^{2}+y^{2} \in \mathbb{R}$ are. Then $f$ is not Lebesgue-integrable 
because $|f|$ is not. Indeed, if the contrary is assumed, then a change to polar coordinates together with Fubini's theorem would imply that the integral $\alpha:=$ $\int_{0}^{2 \pi}\left(\int_{0}^{+\infty}\left|\sin \left(t^{2}\right)\right| \cdot t d t\right) d \theta$ is finite, which is not true because

$$
\begin{aligned}
\alpha & =2 \pi \cdot \int_{0}^{+\infty}\left|\sin \left(t^{2}\right)\right| \cdot t d t \geq 2 \pi \cdot \sum_{n=1}^{\infty} \int_{\left(\frac{\pi}{6}+2 \pi n\right)^{1 / 2}}^{\left(\frac{\pi}{2}+2 \pi n\right)^{1 / 2}}\left|\sin \left(t^{2}\right)\right| \cdot t d t \\
& \geq 2 \pi \cdot \sum_{n=1}^{\infty} \int_{\left(\frac{\pi}{6}+2 \pi n\right)^{1 / 2}}^{\left(\frac{\pi}{2}+2 \pi n\right)^{1 / 2}} \frac{t}{2} d t=\frac{\pi}{2} \cdot \sum_{n=1}^{\infty}\left(\frac{\pi}{2}+2 \pi n-\left(\frac{\pi}{6}+2 \pi n\right)\right) \\
& =\frac{\pi^{2}}{6} \cdot \sum_{n=1}^{\infty} 1=+\infty
\end{aligned}
$$

Now, fix $x_{0} \in \mathbb{R}$. If the function $\varphi: y \in \mathbb{R} \mapsto f\left(x_{0}, y\right)=\sin \left(x_{0}^{2}+y^{2}\right) \in \mathbb{R}$ were Lebesgue-integrable then so would be the function $|\varphi|$. This is, again, false because, if we choose $N \in \mathbb{N}$ large enough to have $x_{0}^{2}<\frac{\pi}{6}+2 \pi N$, then we get

$$
\begin{aligned}
\int_{\mathbb{R}}|\varphi(y)| d y & \geq \int_{\bigcup_{n=N}^{\infty}\left[\left(\frac{\pi}{6}+2 \pi n-x_{0}^{2}\right)^{1 / 2},\left(\frac{\pi}{2}+2 \pi n-x_{0}^{2}\right)^{1 / 2}\right]} \sin \left(x_{0}^{2}+y^{2}\right) d y \\
& \geq \sum_{n=N}^{\infty} \frac{1}{2} \cdot\left(\left(\frac{\pi}{2}+2 \pi n-x_{0}^{2}\right)^{1 / 2}-\left(\frac{\pi}{6}+2 \pi n-x_{0}^{2}\right)^{1 / 2}\right) \\
& =\frac{\pi}{6} \cdot \sum_{n=N}^{\infty} \frac{1}{\left(\frac{\pi}{2}+2 \pi n-x_{0}^{2}\right)^{1 / 2}+\left(\frac{\pi}{6}+2 \pi n-x_{0}^{2}\right)^{1 / 2}}=+\infty
\end{aligned}
$$

by the comparison test and the divergence of $\sum \frac{1}{n^{1 / 2}}$. Therefore, no function $y \mapsto f\left(x_{0}, y\right)$ (and, analogously, no function $\left.x \mapsto f\left(x, y_{0}\right)\right)$ is Lebesgueintegrable.

Now, consider the Fresnel integrals (see, e.g., [1, Chapter 7])

$$
S(x)=\int_{0}^{x} \sin \left(t^{2}\right) d t \text { and } C(x)=\int_{0}^{x} \cos \left(t^{2}\right) d t \quad(x \in \mathbb{R}) .
$$

It is known that $\sin \left(t^{2}\right)$ and $\cos \left(t^{2}\right)$ are Riemann-integrable on $\mathbb{R}$ and that, in the Riemann sense, it holds that

$$
\int_{0}^{+\infty} \sin \left(t^{2}\right) d t=\lim _{x \rightarrow+\infty} S(x)=\sqrt{\frac{\pi}{8}}=\lim _{x \rightarrow+\infty} C(x)=\int_{0}^{+\infty} \cos \left(t^{2}\right) d t .
$$


Since $\sin \left(t^{2}\right)$ and $\cos \left(t^{2}\right)$ are even functions, we also have $\int_{-\infty}^{0} \sin \left(t^{2}\right) d t=$ $\sqrt{\frac{\pi}{8}}=\int_{-\infty}^{0} \cos \left(t^{2}\right) d t$. Note that $S$ and $C$ are odd functions, and so $S(x) \rightarrow$ $\sqrt{\frac{\pi}{8}} \leftarrow C(x)$ as $x \rightarrow-\infty$.

Finally, if all the following integrals are considered in the Riemann sense, we have

$$
\begin{aligned}
\int_{-\infty}^{\infty} & \left(\int_{-\infty}^{\infty} f(x, y) d x\right) d y=\int_{-\infty}^{\infty}\left(\int_{-\infty}^{\infty} \sin \left(x^{2}+y^{2}\right) d x\right) d y \\
= & \int_{-\infty}^{\infty}\left(\int_{-\infty}^{\infty}\left(\sin \left(x^{2}\right) \cos \left(y^{2}\right)+\cos \left(x^{2}\right) \sin \left(y^{2}\right)\right) d x\right) d y \\
= & \int_{-\infty}^{\infty}\left(\int_{-\infty}^{\infty} \sin \left(x^{2}\right) d x\right) \cos \left(y^{2}\right) d y \\
& +\int_{-\infty}^{\infty}\left(\int_{-\infty}^{\infty} \cos \left(x^{2}\right) d x\right) \sin \left(y^{2}\right) d y \\
= & 2 \sqrt{\frac{\pi}{8}} \cdot \int_{-\infty}^{\infty} \cos \left(y^{2}\right) d y+2 \sqrt{\frac{\pi}{8}} \cdot \int_{-\infty}^{\infty} \sin \left(y^{2}\right) d y \\
= & 2 \sqrt{\frac{\pi}{8}} \cdot 2 \sqrt{\frac{\pi}{8}}+2 \sqrt{\frac{\pi}{8}} \cdot 2 \sqrt{\frac{\pi}{8}}=\pi .
\end{aligned}
$$

Since $f(x, y)=f(y, x)$ for all $(x, y) \in \mathbb{R}^{2}$, we derive that the value of the other iterated integral $\int_{-\infty}^{\infty}\left(\int_{-\infty}^{\infty} f(x, y) d y\right) d x$ is also $\pi$. Hence both integrals coincide.

Funding Open Access funding provided thanks to the CRUE-CSIC agreement with Springer Nature.

Open Access. This article is licensed under a Creative Commons Attribution 4.0 International License, which permits use, sharing, adaptation, distribution and reproduction in any medium or format, as long as you give appropriate credit to the original author(s) and the source, provide a link to the Creative Commons licence, and indicate if changes were made. The images or other third party material in this article are included in the article's Creative Commons licence, unless indicated otherwise in a credit line to the material. If material is not included in the article's Creative Commons licence and your intended use is not permitted by statutory regulation or exceeds the permitted use, you will need to obtain permission directly from the copyright holder. To view a copy of this licence, visit http://creativecommons. org/licenses/by/4.0/.

\section{References}

[1] Abramowitz, M., Stegun, I.E. (eds.): Handbook of Mathematical Functions with Formulas, Graphs, and Mathematical Tables. Dover, New York (1972) 
[2] Aron, R., Bernal-González, L., Pellegrino, D., Seoane-Sepúlveda, J.B.: Lineability: The search for linearity in Mathematics, Monographs and Research Notes in Mathematics. Chapman \& Hall/CRC, Boca Raton, FL (2016)

[3] Bernal-González, L., Calderón-Moreno, M. C.: Anti-Fubini and pseudo-Fubini functions, Rev. Real Acad. Cien. Ex. Fís. Nat. Ser. A Mat., 115:127, (2021), 16 pp

[4] Cartan, H.: Théorie élémentaire des fonctions analytiques d'une ou plusieurs variables complexes, 6. Hermann, Paris (1997)

[5] Evans, L.C., Gariepy, R.F.: Measure theory and fine properties of functions. CRC Press, Taylor and Francis, Boca Raton, FL (2015)

[6] Horváth, J.: Topological Vector Spaces and Distributions (Vol. I). AddisonWesley, Reading, MA (1966)

[7] Narici, L., Beckenstein, L.: Topological Vector Spaces, 2nd edn. CRC Press, Chapman and Hall, Boca Raton, FL (2011)

[8] Nielsen, O.A.: An Introduction to Integration and Measure Theory, Canadian Mathematical Society Series of Monographs and Advanced Texts. Wiley, New York (1997)

Luis Bernal-González and María del Carmen Calderón-Moreno

Departamento de Análisis Matemático, Facultad

de Matemáticas, Instituto de Matemáticas Antonio de Castro Brzezicki (IMUS)

Universidad de Sevilla

Avenida Reina Mercedes

Sevilla 41012

Spain

e-mail: lbernal@us.es;

mccm@us.es

Andreas Jung

Fachbereich IV Mathematik

Universität Trier

D-54286 Trier

Germany

e-mail: andreas.tibor.jung@gmail.com

Received: April 4, 2021.

Accepted: November 21, 2021.

Publisher's Note Springer Nature remains neutral with regard to jurisdictional claims in published maps and institutional affiliations. 